\title{
FACTORES TOPOGRÁFICOS Y EDÁFICOS QUE INFLUYEN EN LA ESTRUCTURA DE ESPECIES PERENNES DE ISLAS DE LA costa de Sinaloa, MÉxico
}

\author{
Bardo Heleodoro Sánchez-Soto ${ }^{1,2}$, Edmundo García-Moya ${ }^{1,3}$, Álvaro Reyes-Olivas², \\ Angélica Romero-Manzanares ${ }^{1}$ y Mario Luna-Cavazos ${ }^{1}$. \\ ${ }^{1}$ Colegio de Postgraduados. Texcoco, Estado de México, México, \\ ${ }^{2}$ Facultad de Agricultura del Valle del Fuerte, Universidad Autónoma de Sinaloa. \\ Ciudad de Juan José Ríos, Ahome, Sinaloa, México. \\ ${ }^{3}$ Autor para la correspondencia: edmundo@colpos.mx
}

\begin{abstract}
Resumen: Se tomaron datos de cobertura vegetal de 29 especies perennes y 25 variables del medio en 57 sitios, para investigar la relación vegetación-ambiente en 16 islas de la costa de Sinaloa, México. Los sitios de vegetación se clasificaron con el análisis de conglomerados y se ordenaron con el análisis de correspondencia linearizado (ACL), luego se interpretaron los gradientes ecológicos con el análisis de correspondencia canónica (ACC). Las correlaciones para los datos de especies-medio fueron significativas $(P<0.05)$ para diez variables ambientales. El primer eje estuvo relacionado con nitratos $(0.776)$ y $\mathrm{pH}(-0.584)$, mientras que el segundo eje correlacionó con cobertura rocosa (-0.901), profundidad del suelo (0.757), porcentaje de limo (-0.696), pendiente $(-0.685)$ y porcentaje de arena (0.663). Estas variables fueron las que mejor explicaron la estructura de la vegetación isleña sinaloense. El efecto de tales factores es notorio entre grupos de islas: las que tienen aporte de guano de aves marinas, las islas rocosas de origen volcánico y las de barra arenosa.
\end{abstract}

Palabras clave: análisis multivariante, composición florística, estructura de comunidades, sistemas insulares.

\begin{abstract}
Cover data of 29 perennial species and 25 environmental variables, in 57 sites, were used to assess vegetation-environment relationships of 16 islands in the coast of Sinaloa, Mexico. The sites were classified through cluster analysis and ordered using detrended correspondence analysis (DCA), then ecological gradients were analyzed with canonical correspondence analysis (CCA). Correlations, species-environment, were significant $(P<0.05)$ to ten environmental variables. The first axis was related to nitrate $(0.776)$ and $\mathrm{pH}(-0.584)$, while the second axis correlated with rocky cover (-0.901), soil depth (0.757), percentage of silt (-0.696), slope (-0.685), and percentage of sand (0.663). These variables were those that best explained the structure of the Sinaloa island vegetation. The effect of such factors is noticeable between island groups: those with addition of seabird guano, islands with rocks of volcanic origin, and those of sandbar.
\end{abstract}

Key words: floristic composition, island systems, multivariate analysis, structure of communities.

$\mathbf{E}^{1}$ reemplazo de las especies a lo largo de gradientes ambientales es gradual de acuerdo con la hipótesis del continuum (Pausas y Austin, 2001). El análisis multivarido es una técnica exploratoria que ayuda a esclarecer cuáles factores ambientales influyen en la distribución de las especies y en la estructura de la comunidad. Diversos estudios indican que la vegetación suele asociarse con el relieve (McAuliffe, 1994; Valverde et al., 1996), el macroclima (Aguado-Santacruz et al., 1996; Aguado-Santacruz y García-Moya, 1998), la topografía y las propiedades físico-químicas del suelo
(Huerta-Martínez et al., 2004; Abd El-Ghani et al., 2014). También se ha detectado correlación de la vegetación con el material de origen del suelo y con la fertilidad, exposición de ladera, altitud y precipitación pluvial (Fernández-Palacios y de Nicolás, 1995; Otto et al., 2001; Duarte et al., 2005).

El estudio de gradientes ecológicos en islas mexicanas es limitado. Sólo en la isla Patos, de la Bahía de Ohuira, situada al norte de Sinaloa, Reyes-Olivas et al. (2008) han identificado dos gradientes ecológicos asociados con la composición vegetal: (1) un factor de aridez determinado 
por la exposición de ladera, la profundidad del suelo y la cobertura de rocas y (2) un gradiente químico causado por la deposición del guano de aves marinas. En consecuencia, las características ecológicas que favorecen el establecimiento de la vegetación son desconocidas en la mayoría de las islas de la entidad, por lo que en este trabajo se intenta contribuir con este conocimiento y esclarecer, con la ayuda de técnicas multivariantes de clasificación y ordenación, cuáles factores del medio influyen en la composición y estructura de las especies perennes presentes en 16 islas distribuidas en $500 \mathrm{~km}$ de litoral del estado de Sinaloa. En este trabajo se parte de la hipótesis que, por el origen geológico (volcánico o arenoso), fisiográfico y de enriquecimiento biogeoquímico diferencial entre islas por presencia de aves, se esperaría que, el contenido de rocas, la pendiente y los factores edáficos como el $\mathrm{pH}$, influyan en la presencia de las especies perennes.

\section{Materiales y métodos}

Descripción del área de estudio. Sinaloa se ubica en el noroeste de México $\left(22^{\circ} 29^{\prime}, 27^{\circ} 02^{\prime} \mathrm{N} ; 105^{\circ} 23^{\prime}, 109^{\circ} 28^{\prime}\right.$ $\mathrm{O}$; Figura 1). Tiene $608 \mathrm{~km}^{2}$ de superficie insular, $656 \mathrm{~km}$ de litoral y 221 mil 600 ha de lagunas litorales (Gobierno del Estado de Sinaloa, 2007). Existen cerca de 400 cuerpos insulares (Flores-Campaña et al., 2003) localizados en Bahía de Mazatlán, Altata-Ensenada de Pabellones, Complejo Playa Colorada-Bahía de Santa María-La Reforma y el Sistema Navachiste-Macapule o Bahía de Ohuira (Figura 2A).

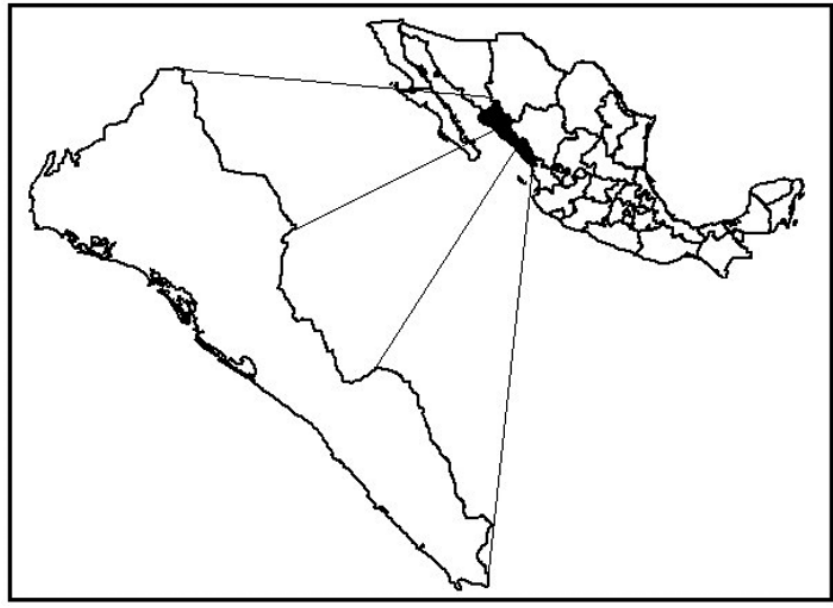

Figura 1. Ubicación de Sinaloa en la República Mexicana.

En la Tabla 1 se presentan las características y ubicación de las islas estudiadas. El clima fue determinado conforme a la nomenclatura descrita por García (1988), mientras que los datos de temperatura y precipitación para cada grupo insular, indicados más abajo, están referenciados con base en las normales climatológicas de algunas estaciones terrestres cercanas, de acuerdo con información de la Comisión Nacional del Agua (CONAGUA, 2014).

La Bahía Mazatlán se sitúa frente al puerto del mismo nombre y las islas muestreadas fueron Pájaros, Venados y Lobos (Figura 2B). El clima es tropical y subtropical con

Tabla 1. Características y ubicación de las 16 islas de la costa de Sinaloa.

\begin{tabular}{|c|c|c|c|c|}
\hline Sistema lagunar & Isla & Área (ha) & $\begin{array}{c}\text { Distancia al } \\
\text { continente (km) }\end{array}$ & $\begin{array}{l}\text { Coordenadas geográficas } \\
\text { (centro de isla) }\end{array}$ \\
\hline \multirow[t]{3}{*}{ Bahía Mazatlán } & Lobos & 10.5 & 1.89 & $23^{\circ} 13^{\prime} 31^{\prime \prime} \mathrm{N}, 106^{\circ} 27^{\prime} 51^{\prime \prime} \mathrm{O}$ \\
\hline & Pájaros & 48 & 0.87 & $23^{\circ} 15^{\prime} 16^{\prime \prime} \mathrm{N}, 106^{\circ} 28^{\prime} 35^{\prime \prime} \mathrm{O}$ \\
\hline & Venados & 54 & 1.23 & $23^{\circ} 14^{\prime} 00^{\prime \prime} \mathrm{N}, 106^{\circ} 28^{\prime} 00^{\prime \prime} \mathrm{O}$ \\
\hline Altata-Ensenada Pabellones & Iguanas & 27.4 & 3.61 & $24^{\circ} 26^{\prime} 16^{\prime \prime} \mathrm{N}, 107^{\circ} 37^{\prime} 33^{\prime \prime} \mathrm{O}$ \\
\hline \multirow{3}{*}{$\begin{array}{l}\text { Complejo Playa Colorada-Bahía } \\
\text { de Santa María-La Reforma }\end{array}$} & Altamura & 8,000 & 3.94 & $24^{\circ} 58^{\prime} 13^{\prime \prime} \mathrm{N}, 108^{\circ} 11^{\prime} 13^{\prime \prime} \mathrm{O}$ \\
\hline & Saliaca & 2,100 & 0.84 & $25^{\circ} 10^{\prime} 07^{\prime \prime} \mathrm{N}, 108^{\circ} 18^{\prime} 51^{\prime \prime} \mathrm{O}$ \\
\hline & Talchichilte & 12,741 & 3.21 & $24^{\circ} 55^{\prime} 02^{\prime \prime} \mathrm{N}, 108^{\circ} 04^{\prime} 39^{\prime \prime} \mathrm{O}$ \\
\hline \multirow[t]{5}{*}{ Navachiste-Macapule } & Alta Blanca/ El Molcajete & 1.8 & 3.21 & $25^{\circ} 18^{\prime} 18^{\prime \prime} \mathrm{N}, 108^{\circ} 52^{\prime} 05^{\prime \prime} \mathrm{O}$ \\
\hline & Cardo & 0.9 & 4.44 & $25^{\circ} 29^{\prime} 11^{\prime \prime} \mathrm{N}, 108^{\circ} 51^{\prime} 05^{\prime \prime} \mathrm{O}$ \\
\hline & Guasayeye & 54 & 5.12 & $25^{\circ} 28^{\prime} 41^{\prime \prime} \mathrm{N}, 108^{\circ} 50^{\prime} 30^{\prime \prime} \mathrm{O}$ \\
\hline & Huitussera & 26 & 1.70 & $25^{\circ} 29^{\prime} 53^{\prime \prime} \mathrm{N}, 108^{\circ} 47^{\prime} 45^{\prime \prime} \mathrm{O}$ \\
\hline & Tesobiate & 510 & 1.14 & $25^{\circ} 29^{\prime} 39^{\prime \prime} \mathrm{N}, 108^{\circ} 49^{\prime} 21^{\prime \prime} \mathrm{O}$ \\
\hline \multirow[t]{4}{*}{ Bahía de Ohuira } & Bledos & 9.87 & 1.12 & $25^{\circ} 36^{\prime} 04^{\prime \prime} \mathrm{N}, 108^{\circ} 59^{\prime} 00^{\prime \prime} \mathrm{O}$ \\
\hline & Tunosa & 0.93 & 1.18 & $25^{\circ} 34^{\prime} 56^{\prime \prime} \mathrm{N}, 109^{\circ} 00^{\prime} 52^{\prime \prime} \mathrm{O}$ \\
\hline & Mazocahui & 20.8 & 0.14 & $25^{\circ} 34^{\prime} 10^{\prime \prime} \mathrm{N}, 109^{\circ} 00^{\prime} 48^{\prime \prime} \mathrm{O}$ \\
\hline & Patos & 23.0 & 1.38 & $25^{\circ} 37^{\prime} 11^{\prime \prime} \mathrm{N}, 109^{\circ} 00^{\prime} 46^{\prime \prime} \mathrm{O}$ \\
\hline
\end{tabular}




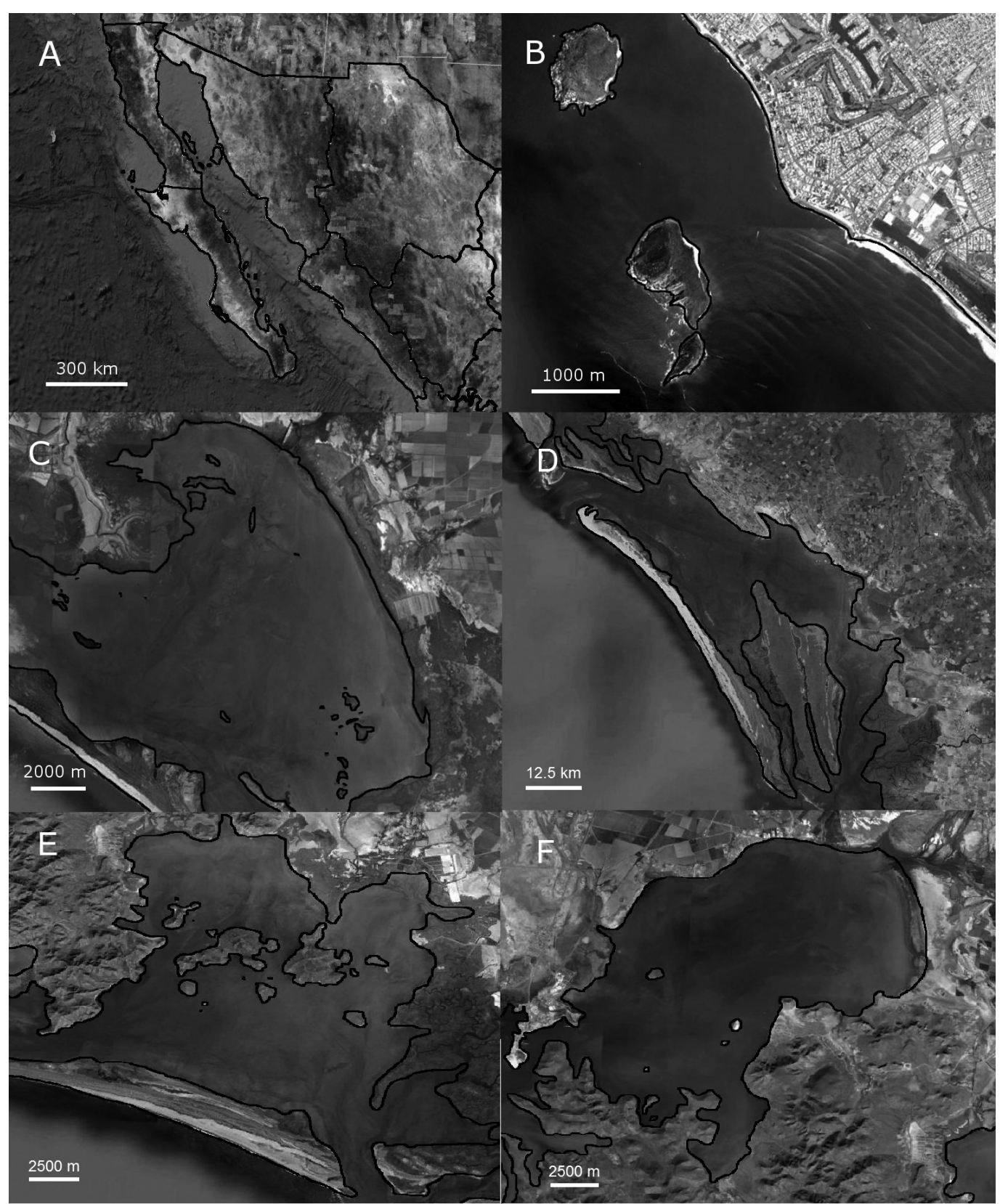

Figura 2. Localización de los sistemas lagunares de Sinaloa. A) Ubicación de la entidad. B) Bahía Mazatlán. C) Altata-Ensenada Pabellones. D) Bahía de Santa María-La Reforma. E) Navachiste-Macapule, F) Bahía de Ohuira.

temperatura media anual de $29.7{ }^{\circ} \mathrm{C}$ y precipitación media anual de 579.9 mm (Estación 25191 CFE, Cuenca Río Presidio). La descripción de la flora y tipos de vegetación de las tres islas la consignan Flores-Campaña et al. (1996) y VegaAviña et al. (2001), quienes destacan la presencia de bosque espinoso, matorral xerófilo, pastizal y vegetación halófita, quienes se basaron para definir estos términos, en la clasificación de la vegetación de Rzedowski (1978). La Bahía Altata-Ensenada Pabellones se localiza en la parte centronorte del estado, donde se localizan islas como Iguanas, El Infiernito y Guamuchilito (Figura 2C); la primera se incluyó en el estudio. El clima es seco semicálido, con precipitación pluvial de $524.0 \mathrm{~mm}$ y temperatura media anual de $23.7^{\circ} \mathrm{C}$ (Estación 25021, San Ignacio CONAGUA-DGE, Cuenca de los Ríos Piaxtla, Elote, Quelite). La vegetación corresponde al matorral espinoso, matorral xerófilo, y vegetación acuática y subacuática. El Complejo Insular Playa Colorada-Bahía de Santa María-La Reforma, es el más grande de la zona centro, cuenta con 53 mil ha y 153 islas; para este estudio se consideraron Altamura, Saliaca y Talchichilte por sus características ecológicas (Figura 2D). La vegetación consiste de halófitas, manglar, matorral xerófilo y pastizal (Flores- 
Tabla 2. Resultados del análisis de correspondencia canónica entre variables del medio y datos de comunidades vegetales de 16 islas de la costa de Sinaloa. Los asteriscos representan la significancia según la prueba de Monte Carlo con $\mathrm{P}<0.05$.

\begin{tabular}{|c|c|c|c|c|}
\hline Ejes & 1 & 2 & 3 & $\begin{array}{c}\text { Inercia } \\
\text { total }\end{array}$ \\
\hline Valor propio & $0.570^{*}$ & $0.499^{*}$ & $0.333^{*}$ & 4.3540 \\
\hline \multicolumn{5}{|l|}{ Varianza/Datos/Especies } \\
\hline Variación explicada (\%) & 13.1 & 11.5 & 7.7 & \\
\hline $\begin{array}{l}\text { Varianza explicada } \\
\text { acumulada (\%) }\end{array}$ & 13.1 & 24.6 & 32.3 & \\
\hline Correlación Especies-Ambiente & $0.863^{*}$ & $0.959^{*}$ & $0.855^{*}$ & \\
\hline \multicolumn{5}{|l|}{ Correlaciones de variables con } \\
\hline \multicolumn{5}{|l|}{ los ejes } \\
\hline $\mathrm{pH}$ & $-0.584^{*}$ & 0.400 & -0.297 & \\
\hline Ca (meq/ L) & -0.019 & -0.574 & 0.116 & \\
\hline K (meq/ L) & 0.040 & -0.512 & $0.680^{*}$ & \\
\hline HCO3 (meq/ L) & -0.188 & -0.471 & -0.092 & \\
\hline Arena $(\%)$ & 0.257 & $0.663^{*}$ & 0.451 & \\
\hline Limo (\%) & -0.113 & $-0.696^{*}$ & -0.273 & \\
\hline Arcilla (\%) & -0.066 & -0.571 & -0.488 & \\
\hline Materia orgánica (\%) "MO" & 0.290 & -0.560 & -0.125 & \\
\hline Nitratos (ppm) & $0.776^{*}$ & -0.246 & 0.095 & \\
\hline Fosfatos (ppm) "P" & -0.030 & -0.533 & 0.497 & \\
\hline Profundidad del suelo $(\mathrm{cm})$ "Prof" & -0.079 & $0.757^{*}$ & 0.368 & \\
\hline Cobertura rocosa $(\%)$ "CR" & 0.144 & $-0.901 *$ & -0.346 & \\
\hline Pendiente $\left({ }^{\circ}\right)$ "Pen" & 0.211 & $-0.685^{*}$ & -0.302 & \\
\hline Índice de Radiación Solar (\%) & -0.362 & 0.124 & 0.066 & \\
\hline Índice de Calor & -0.218 & -0.013 & 0.168 & \\
\hline
\end{tabular}

Campaña et al., 2003). En el sistema Navachiste-Macapule existen 18 cuerpos insulares, de los cuales se eligieron Alta Blanca, Cardo, Guasayeye, Huitussera y Tesobiate (Figura 2E). La vegetación consiste de bosque espinoso, manglar, matorral xerófito, pastizal, vegetación de dunas costeras y vegetación halófita (Díaz, 2008). La Bahía de Ohuira tiene seis islas, de ellas se estudiaron Bledos, Mazocahui I y Patos (Figura 2F). El clima es muy seco y cálido, con precipitación media anual de $369.7 \mathrm{~mm}$ y temperatura promedio de $24.3{ }^{\circ} \mathrm{C}$ (Estación 25013 CONAGUA-DGE, Cuenca Bahía de Ohuira Navachiste). El tipo de vegetación se caracteriza por ser matorral crasicaule (Reyes-Olivas, 2002; Reyes-Olivas et al., 2008).

Relación vegetación medio. De agosto de 2007 a agosto 2009 se realizaron registros de variables topográficas, edáficas y cobertura vegetal de las especies perennes en 16 islas continentales de Sinaloa. Los sitios de muestreo en cada isla fueron seleccionados por exposición, expresada en grados azimut (Brújula Brunton Tipo 15): este (46-135 ), sur (135$\left.225^{\circ}\right)$, oeste $\left(226-315^{\circ}\right)$, norte $\left(316-45^{\circ}\right)$ y cima (planicie superior), donde también se registró la pendiente del terre- no en grados, con un nivel de mano Abney, la cobertura de piedras (\%) por intercepción lineal a lo largo de $10 \mathrm{~m}$, y se midió la profundidad del suelo $(\mathrm{cm})$ con una barrena tipo tornillo. El Índice de Radiación Solar (IRS), fue estimado para el 22 de diciembre, a las 12 h, con una declinación solar de $-23.45^{\circ}$ y la localización en grados de casa isla, datos que son utilizados en la fórmula tomada de Oke (1987): IRS = $\operatorname{Cos} \theta$, mientras que el $\operatorname{Cos} \theta=\operatorname{Cos} \beta \operatorname{Cos} \theta+\operatorname{Sen} \Omega$ Sen $\mathrm{Z} \operatorname{Cos}(\Omega-\vartheta)$, donde $\Omega=$ ángulo del azimut solar $\left(180^{\circ}\right)$, $\vartheta=$ azimut de la pendiente (de acuerdo con la exposición del sitio muestreado, norte, este, oeste, sur o cima). También se calculó un índice de calor (IC) (Geiger, 1966): IC = Cos (exposición - $225 \times \tan$ (pendiente). La cobertura vegetal se

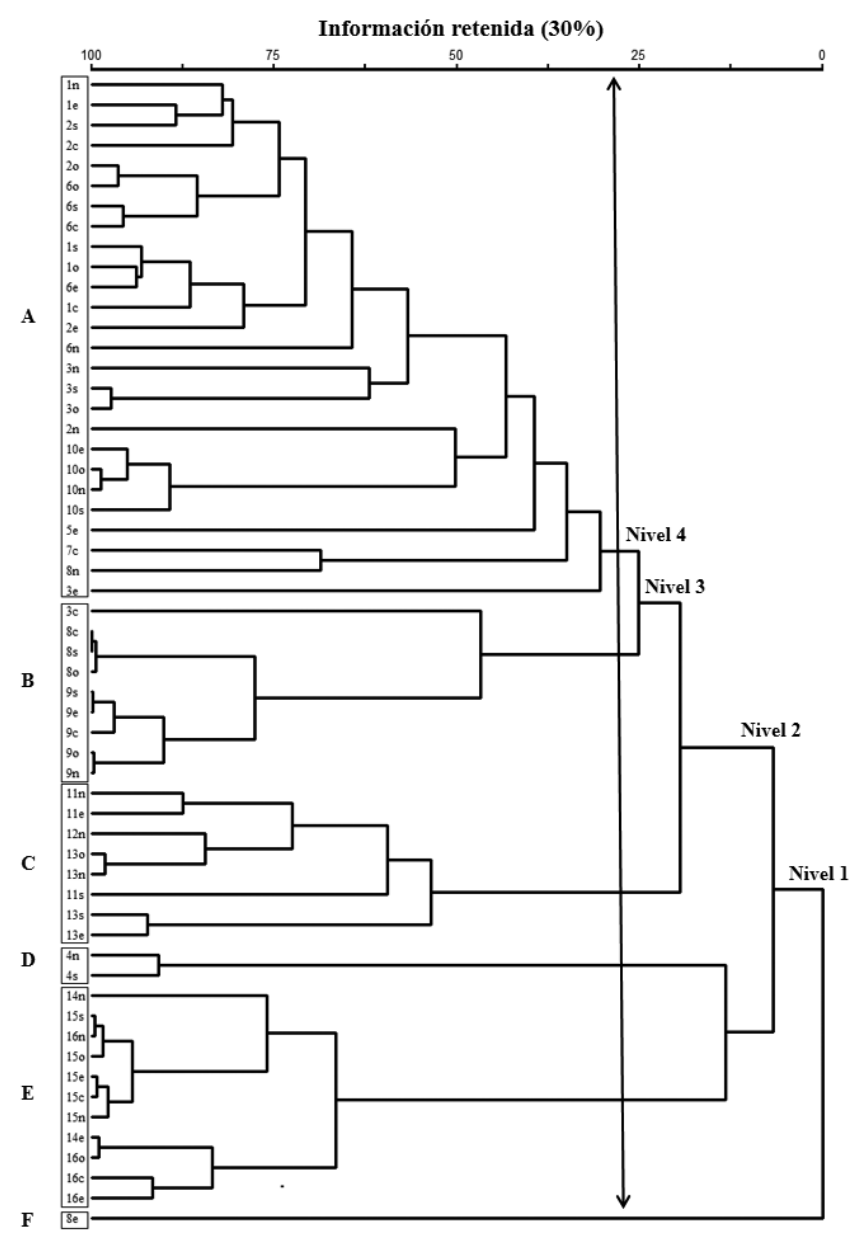

Figura 3. Dendrograma de los agrupamientos de 57 sitios de muestreo de especies perennes de 16 islas de la costa de Sinaloa. Al cuarto nivel se tiene un corte menor de $30 \%$ de la información retenida. Los nombres corresponden a sitios de muestreo definidos por número y letra. El número pertenece al nombre de la isla (1 = Tesobiate, 2 = Guasayeye, $3=$ Huitussera, $4=$ Alta Blanca, $5=$ Cardo, $6=$ Mazocahui, $7=$ Tunosa, $8=$ Bledos, $9=$ Patos, $10=$ Tal chichilte, 11 = Saliaca, $12=$ Altamura, $13=$ Iguanas, $14=$ Venados, 15 = Pájaros y $16=$ Lobos) y la letra describe su localización $(\mathrm{n}=$ norte, $\mathrm{e}=$ este, $\mathrm{s}=$ sur, $\mathrm{o}=$ oeste $\mathrm{y} \mathrm{c}=$ cima). 
determinó por línea interceptriz (Canfield, 1941), mediante dos líneas perpendiculares, de $50 \mathrm{~m}$ cada una.

En cada sitio de muestreo se recolectaron muestras de suelo de cinco a $15 \mathrm{~cm}$ de profundidad para determinar 19 variables físico-químicas: textura del suelo (porcentaje de arena, limo y arcilla), conductividad eléctrica $\left(\mathrm{dS} \mathrm{m} \mathrm{m}^{-1}\right), \mathrm{pH}$, porcentaje de sodio intercambiable (PSI), razón de adsorción de sodio (RAS), cationes en meq/ $100 \mathrm{~g}\left(\mathrm{Ca}^{++}, \mathrm{Mg}^{++}, \mathrm{Na}^{+}\right.$, $\left.\mathrm{K}^{+}\right)$, aniones en meq/ $100 \mathrm{~g}\left(\mathrm{HCO}_{3^{-}}, \mathrm{Cl}-, \mathrm{SO}_{4}=\right)$, materia orgánica (\%), nitratros (ppm) y fosfatos (ppm) de acuerdo con los métodos descritos por Walkley y Black (1934), Richards (1954), Bremner (1965), Day (1965) y CSTPA (1980). Además, se recolectaron ejemplares de las especies, las que se depositaron en el Herbario de la Facultad de Agricultura del Valle del Fuerte, Universidad Autónoma de Sinaloa en Juan José Ríos, Ahome, Sinaloa. La identidad taxonómica se determinó con base en floras regionales, entre ellas las obras de Shreve y Wiggins (1964) y Wiggins (1980), y los nombres y autores de las especies se cotejaron en la base de datos Tropicos (http://www.tropicos.org/ consultado 15 de noviembre, 2014). La matriz final de coberturas vegetales incluyó 29 especies perennes y 57 sitios de muestreo; la matriz secundaria (matriz ambiental) integró 23 variables del mismo número de sitios de vegetación. Con la primera matriz se realizó un análisis de agrupamiento entre las 16 islas; el propósito fue analizar la semejanza en composición florística entre las unidades de muestreo. Se eligió el Índice de Sørensen como el coeficiente de distancia entre grupos, por ser robusto y porque es una de las medidas de similitud más efectivas entre muestras o especies (McCune y Grace, 2002; McCune y Mefford, 2011). Los grupos se integraron por el método de ligamiento promedio (UPGMA). Es importante señalar que las coberturas vegetales se transformaron con raíz cuadrada para los análisis de clasificación y ordenación con la finalidad de estandarizar los datos. Para la ordenación de la vegetación se utilizó el análisis de correspondencia linearizado (ACL) y el análisis de correspondencia canónica (ACC), ter Braak (1986). Estas técnicas son adecuadas cuando las especies muestran relaciones de tipo unimodal a gradientes ambientales (ter Braak y Prentice, 1988). La prueba de permutaciones de Monte Carlo asegura que la variación explicada en los ejes de ordenación sea producto de las variables consideradas y no de efectos aleatorios (Palmer, 1993). Para evitar la multicolinealidad entre las 25 variables ambientales, éstas fueron sometidas a la prueba de correlación con el coeficiente de Pearson en el paquete estadístico SAS (SAS Institute, 2009); las variables que presentaron una $r<0.7$ y $P>0.05$ fueron eliminadas, éstas fueron: conductividad eléctrica, porcentaje de sodio, razón de adsorción de sodio, magnesio, cloro, sulfato y exposición de ladera. Las 15 variables útiles fueron las indicadas más adelante, en la Tabla 2 de resultados. Los análisis de clasificación y ordenación se realizaron con los programas CANOCO versión 4.5 y PC-ORD ver. 6.0 (ter Braak y Šmilauer, 2002; McCune y Mefford, 2011).

\section{Resultados}

Composición de especies perennes y cobertura vegetal. Se registraron 29 especies agrupadas en 15 familias botánicas. Destacaron tres familias por agrupar a 17 de las 29 especies (58.6 \%): Euphorbiaceae (7 especies), Cactaceae (5) y Fabaceae (5). Existe una cobertura vegetal promedio de $72.7 \pm$ $44.3 \%$ en los 57 sitios muestreados; Bursera laxiflora contribuye con $11.6 \%$ y ocurre en el mayor número de sitios (31), en cambio Cylindropuntia thurberi registra la menor cobertura $0.3 \%$, en seis sitios (Tabla 3 ).

Clasificación. La interpretación de los agrupamientos se consideró al cuarto nivel del dendrograma, que equivale a un corte menor de $30 \%$ de la información retenida, observándose cinco grupos de islas y un sitio separado del resto (Figura 3). Esto es coherente con los grupos formados por la ordenación de los sitios y las especies obtenidos en el ACL (Figura 4); los tres primeros ejes explican el $35 \%$ de la varianza total de las especies; el elevado valor propio obtenido de $\lambda=0.793$ para el primer eje, indica una alta correlación entre los sitios y las especies, mientras que la longitud del gradiente es de 4.835, que indica una amplia diferencia en la composición de especies entre los sitios de las islas (Hill, 1979; Hill y Gauch, 1980), definido por gradientes ambientales relacionados con el eje 1; para el eje 2, el valor propio es de 0.428 con una longitud del gradiente de 3.619.

El grupo A, en el dendograma está compuesto por 26 sitios de ocho islas de tres sistemas lagunares; Bledos (8n), Mazocahui (6n, 6e, 6s, 6o y 6c) y Tunosa (7c) de bahía de Ohuira; Cardo (5e), Guasayeye (2n, 2e, 2s, 2o y 2c), Huitussera (3n, 3e, 3s y 3o) y Tesobiate (1n, 1e, 1s, 1o y 1c) de Navachiste, y Talchichilte (10n, 10e, 10s y 10o) de Santa María, mientras que en el ACL, salen los sitios 10 n y 10 o de Talchichilte y se incorporan 8e de Bledos y $3 \mathrm{c}$ de Huitussera. Las especies más comunes en este grupo de sitios son Bursera laxiflora, Euphorbia californica, Jatropha cinerea, Stenocereus thurberi var. thurberi, Agave angustifolia, Opuntia spp., Jatropha cordata, J. cuneata y Coursetia glandulosa. El grupo B, se integra por nueve sitios de Bledos $(8 \mathrm{~s}, 8$ o y $8 \mathrm{c})$ y Patos $(9 \mathrm{n}, 9 \mathrm{e}, 9 \mathrm{~s}, 9$ o y $9 \mathrm{c})$ de bahía de Ohuira más el sitio $3 c$ de Huitussera, bahía de Navachiste; en las dos primeras predomina Opuntia spp., mientras que en la tercera son Croton fragilis, Haematoxylum brasiletto y Melochia pyramidata. El grupo C, está representado por ocho sitios exclusivos de islas de Ensenada Pabellones, en las cuales la especie común es Lycium richii y J. cinerea es una especie frecuente de estos sitios; Altamura, tiene a J. cinerea y Jacquinia pungens como especies importantes; Iguanas a Crateva tapia, mientras que en Saliaca J. cinerea es muy frecuente. El grupo D, se define por las localidades 4n y 4s de Alta Blanca, Bahía de Navachiste y Stenocereus alamosensis es la especie que predomina. El grupo E, comprende las 11 unidades de muestreo de las islas de Bahía 
Bardo Heleodoro Sánchez-Soto et al.

Tabla 3. Cobertura vegetal de las especies perennes agrupadas por familia, registradas en las unidades de muestreo y acrónimos utilizados en el análisis de clasificación y ordenación con PC-ORD versión 6.0 y CANOCO versión 4.5 (ter Braak y Šmilauer, 2002; McCune y Mefford, 2011).

\begin{tabular}{|c|c|c|c|c|}
\hline Familia & Acrónimo & Nombre científico & No. Sitios & $\begin{array}{c}\text { Cobertura } \\
\text { vegetal }(\%)\end{array}$ \\
\hline Agavaceae & Aga ang & Agave angustifolia Haw. & 21 & 3.10 \\
\hline Apocynaceae & Plu rub & Plumeria rubra fo. acutifolia (Poir.) Woodson & 9 & 6.60 \\
\hline Burseraceae & Bur lax & Bursera laxiflora S. Watson & 31 & 11.60 \\
\hline \multirow[t]{5}{*}{ Cactaceae } & Cyl thu & Cylindropuntia thurberi (Engelm.) F.M. Knuth & 6 & 0.30 \\
\hline & Opu spp & Opuntia spp. & 22 & 5.00 \\
\hline & Pac pec & Pachycereus pecten-aboriginum (Engelm. ex S. Watson) Britton \& Rose & 15 & 1.30 \\
\hline & Ste ala & Stenocereus alamosensis (J.M.Coult.) A.C.Gibson \& K.E. Horak & 13 & 0.40 \\
\hline & Ste thu & Stenocereus thurberi (Engelm.) Buxb. var. thurberi & 16 & 1.30 \\
\hline Capparaceae & Cra tap & Crateva tapia L. & 15 & 4.50 \\
\hline Convolvulaceae & Ipo arb & Ipomoea arborescens (Humb. \& Bonpl. ex Willd.) G. Don & 11 & 2.00 \\
\hline \multirow[t]{7}{*}{ Euphorbiaceae } & Cro fra & Croton fragilis Kunth & 7 & 0.70 \\
\hline & Cro pun & Croton punctatus Jacq. & 6 & 2.50 \\
\hline & Eup cal & Euphorbia californica Benth. & 23 & 5.10 \\
\hline & Eup tom & Euphorbia tomentulosa S. Watson & 6 & 0.40 \\
\hline & Jat cin & Jatropha cinerea (Ortega) Müll. Arg. & 22 & 7.20 \\
\hline & Jat cor & Jatropha cordata (Ortega) Müll. Arg. & 11 & 1.10 \\
\hline & Jat cun & Jatropha cuneata Wiggins \& Rollins & 11 & 1.90 \\
\hline \multirow[t]{5}{*}{ Fabaceae } & Cae pla & Caesalpinia platyloba S. Watson & 6 & 3.20 \\
\hline & Cou gla & Coursetia glandulosa A. Gray & 10 & 1.90 \\
\hline & Des cov & Desmanthus covillei (Britton \& Rose) Wiggins & 6 & 1.00 \\
\hline & Hae bra & Haematoxylum brasiletto $\mathrm{H}$. Karst. & 7 & 1.50 \\
\hline & Lys div & Lysiloma divaricatum (Jacq.) J.F. Macbr. & 9 & 0.70 \\
\hline Moraceae & Fic pet & Ficus petiolaris Kunth & 11 & 3.00 \\
\hline Rhamnaceae & Kar hum & Karwinskia humboldtiana (Schult.) Zucc. & 8 & 0.80 \\
\hline Sapindaceae & Pau son & Paullinia sonorensis S. Watson & 6 & 0.80 \\
\hline Solanaceae & Lyc ric & Lycium richii A. Gray & 12 & 1.90 \\
\hline Sterculiaceae & Mel pyr & Melochia pyramidata L. & 7 & 0.90 \\
\hline Theophrastaceae & Jac pun & Jacquinia pungens A. Gray & 8 & 1.20 \\
\hline Verbenaceae & Lan cam & Lantana camara L. & 7 & 0.80 \\
\hline
\end{tabular}

Mazatlán; Venados (14n y 14e), Pájaros (15n, 15e, 15s, 15o y 15c) y Lobos (16n, 16e, 16o y 16c); en ellas Ficus petiolaris es la especie común Plumeria rubra fo. acutifolia y Crateva tapia son especies muy frecuentes en este conjunto de islas; otras especies importantes son Agave angustifolia y Stenocereus alamosensis.

Ordenación indirecta. La asociación de los sitios obtenidos en la clasificación es coherente con los grupos formados por la ordenación de los sitios y las especies obtenidos en el ACL (Figura 4); los tres primeros ejes explican el $35 \%$ de la varianza total de las especies; el elevado valor propio obtenido de $\lambda=0.793$ para el primer eje indica una alta correlación entre los sitios y las especies, mientras que la longitud del gradiente es de 4.835, una amplia diferencia en la composición de especies entre los sitios de las islas (Hill, 1979; Hill y Gauch, 1980), definido por gradientes ambientales relacionados con el eje 1; para el eje 2, el valor propio es de 0.428 con una longitud del gradiente de 3.619 .

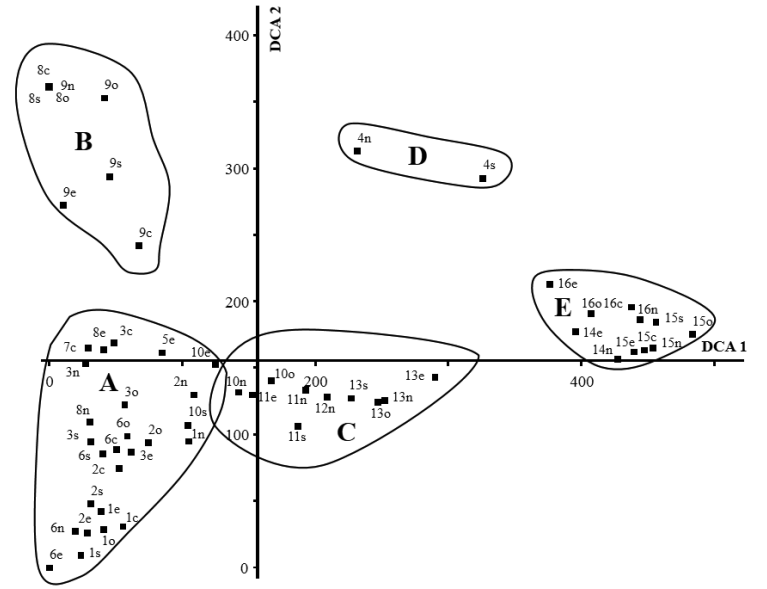

Figura 4. Diagrama de ordenación de 57 sitios de muestreo, en los dos primeros ejes del análisis de correspondencia linearizado (ACL) de 57 sitios de muestreo con 29 especies perennes, incluidos en 16 islas de la costa de Sinaloa. Las abreviaturas de los 57 sitios son las mismas que en la Figura 2. 
La mayoría de las islas del grupo A son pedregosas $(51.16 \%)$, excepto los sitios $10 \mathrm{n}$ y 10 o de Talchichilte $(1 \%)$. Los suelos son de textura franca o franca arenosa de 16.58 $\pm 19.17 \mathrm{~cm}$ de profundidad con $\mathrm{pH}$ de $6.13 \pm 0.64$ existen bajos aportes de nitratos $(15.75 \pm 14.67 \mathrm{ppm})$ pero altos de fosfatos (186.70 $\pm 457.64 \mathrm{ppm})$, principalmente en los sitios $8 \mathrm{n}$ y $8 \mathrm{e}$ de Bledos, $7 \mathrm{c}$ de Tunosa, $5 \mathrm{e}$ de Cardo y $6 \mathrm{c}$ de Mazocahui, sitios que sirven de refugio, percha o reproducción de aves marinas o terrestres. En el ACL, el grupo B está formado por ocho de los nueve sitios derivados del análisis de clasificación, excepto el sitio 3c de Huitussera; se incluyeron islas rocosas $(57.19 \%)$ de suelo profundo (21.66 \pm $13.41 \mathrm{~cm})$, ricos en materia orgánica $(5.30 \pm 2.43 \%)$ con $\mathrm{pH}$ ácido (4.95 \pm 0.81$)$, caracterizados por la presencia de grandes colonias de aves que producen guano, producto que incrementa cationes (374.76 ppm), aniones (216.48 ppm) y fosfatos $(3091.30 \pm 865.79 \mathrm{ppm})$ en el suelo. En el ACL, a los sitios del grupo C se integran los sitios 10n y 10o de Talchichilte con Jatropha cinerea y Croton punctatus. Las islas son arenosas (79.56 $\pm 14.07 \%)$, el suelo tiene un $\mathrm{pH}$ de $6.85 \pm 0.92$, bajo contenido de materia orgánica $1.03 \pm 0.6 \%$ y profundidad mayor a los $65 \pm 14.14 \mathrm{~cm}$. El grupo D está conformado por islas rocosas $(62.80 \%)$ con suelos someros $(2.05 \pm 2.75 \mathrm{~cm})$ de $\mathrm{pH}$ ácido $(5.00 \pm 0.28)$, e ingresos de guano que incrementan los fosfatos $(2,871.65 \pm 4,057.59$ $\mathrm{ppm})$. El grupo $\mathrm{E}$ incluye islas que tienen cobertura de rocas de $50 \%$ con suelos profundos $(13.46 \pm 11.91 \mathrm{~cm})$, ricos en materia orgánica $(4.76 \pm 2.40 \%)$ y pH ácido de $5.38 \pm 0.80$; destacan por la presencia de aves como la garza gris ( $\mathrm{Pla}$ talea ajaja L.) y el pelícano café (Pelecanus occidentalis L.) que aportan guano. En la isla Pájaros, el guano eleva los nitratos de 1,660.2 a 4,477.1 ppm.

Ordenación directa. El ACC que relacionó la comunidad vegetal con las variables ambientales, reveló que los tres ejes de ordenación explican el $32.3 \%$ de la variación total de las especies. Las correlaciones para los datos de especies-medio son significativas $(P<0.05)$ para diez variables ambientales (Tabla 2). El primer eje es un gradiente relacionado con nitratos (0.776) y $\mathrm{pH}(-0.584)$, en cambio el segundo eje se correlaciona con cobertura rocosa $(-0.901)$, profundidad del suelo (0.757), porcentaje de limo (-0.696), pendiente $(-0.685)$ y porcentaje de arena $(0.663)$.

La relación de los patrones geográficos de las especies vegetales en las islas con las variables ambientales se infiere de la Figura 5. Los nitratos (eje 1) en promedio alcanzan valores de 3,042 ppm en los sitios de la isla Pájaros, en donde habitan plantas como Crateva tapia, Ficus petiolaris, Plumeria rubra fo. acutifolia y Stenocereus alamosensis. La correlación de fosfatos con el eje 2, muestra concentraciones máximas de 3,504 ppm en la isla Patos, donde domina Opuntia spp., mientras que los sitios con menor fosfato ( 0.43 $\mathrm{ppm}$ ) ocurren en Saliaca, un lugar carente de nopales. Los sitios de Altamura y Saliaca se correlacionan con pH (7.3

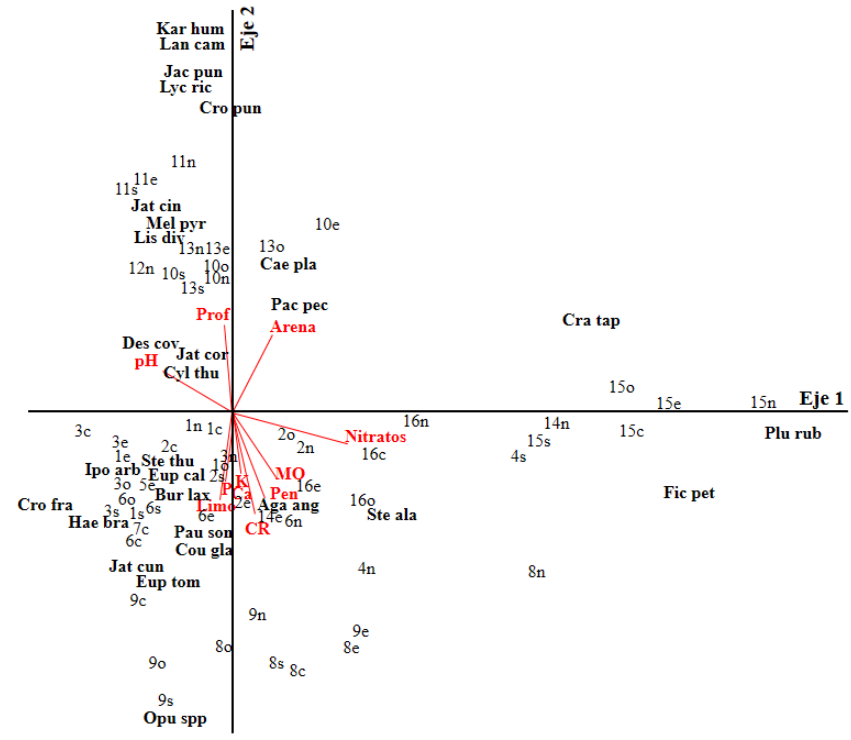

Figura 5. Diagrama de ordenación de los sitios de muestreo y especies perennes con base en el análisis de correspondencia canónica (ACC). Las abreviaturas de los 57 sitios son las mismas que en la Figura 3, mientras que los acrónimos de las 29 especies se indican en el Cuadro 1 . Arena $=$ porcentaje de arena, Limo = porcentaje de limo, $\mathrm{CR}=$ cobertura rocosa, Pen $=$ pendiente del suelo, Prof $=$ profundidad del suelo, $\mathrm{MO}=$ materia orgánica, $\mathrm{pH}=\mathrm{pH}$ del suelo,

$\mathrm{Ca}=$ Calcio, $\mathrm{K}=$ Potasio, Nitratos $=$ Nitratos y $\mathrm{P}=$ Fosfatos .

y 7.7), mientras que la profundidad del suelo es mayor en los sitios de Altamura, Iguanas, Saliaca y Talchichilte, que se caracterizan por ser islas arenosas con profundidad de más de $100 \mathrm{~cm}$, cubiertas por Caesalpinia platyloba, Croton punctatus, Jacquinia pungens, Jatropha cinerea, Karwinskia humboldtiana, Lantana camara y Lycium richii.

La ordenación de la mayoría de los sitios y de las especies se da en la parte central de la Figura 5. Los sitios corresponden a islas que destacan por ser rocosas y se ubican en tres sistemas lagunares: en Bahía de Ohuira son Mazocahui y Tunosa; en Navachiste: Cardo, Guasayeye, Huitussera y Tesobiate; por último, en Bahía Mazatlán, islas Venados y Lobos. Las especies que principalmente habitan las islas rocosas mencionadas son: Agave angustifolia, Bursera laxiflora, Coursetia glandulosa, Croton fragilis, Euphorbia californica, E. tomentulosa, Haematoxylum brasiletto, Ipomoea arborescens, Jatropha cuneata, Paullinia sonorensis y Stenocereus thurberi var. thurberi.

\section{Discusión}

Los resultados demuestran que las condiciones topográficas y edáficas afectan la estructura vegetal en las islas de la costa de Sinaloa, factores que también son importantes en la distribución de especies de plantas, en los desiertos Sonorense y Chihuahuense del norte mexicano (Parker, 1991; McAuliffe, 1994; Valverde et al., 1996) y en ambientes se- 


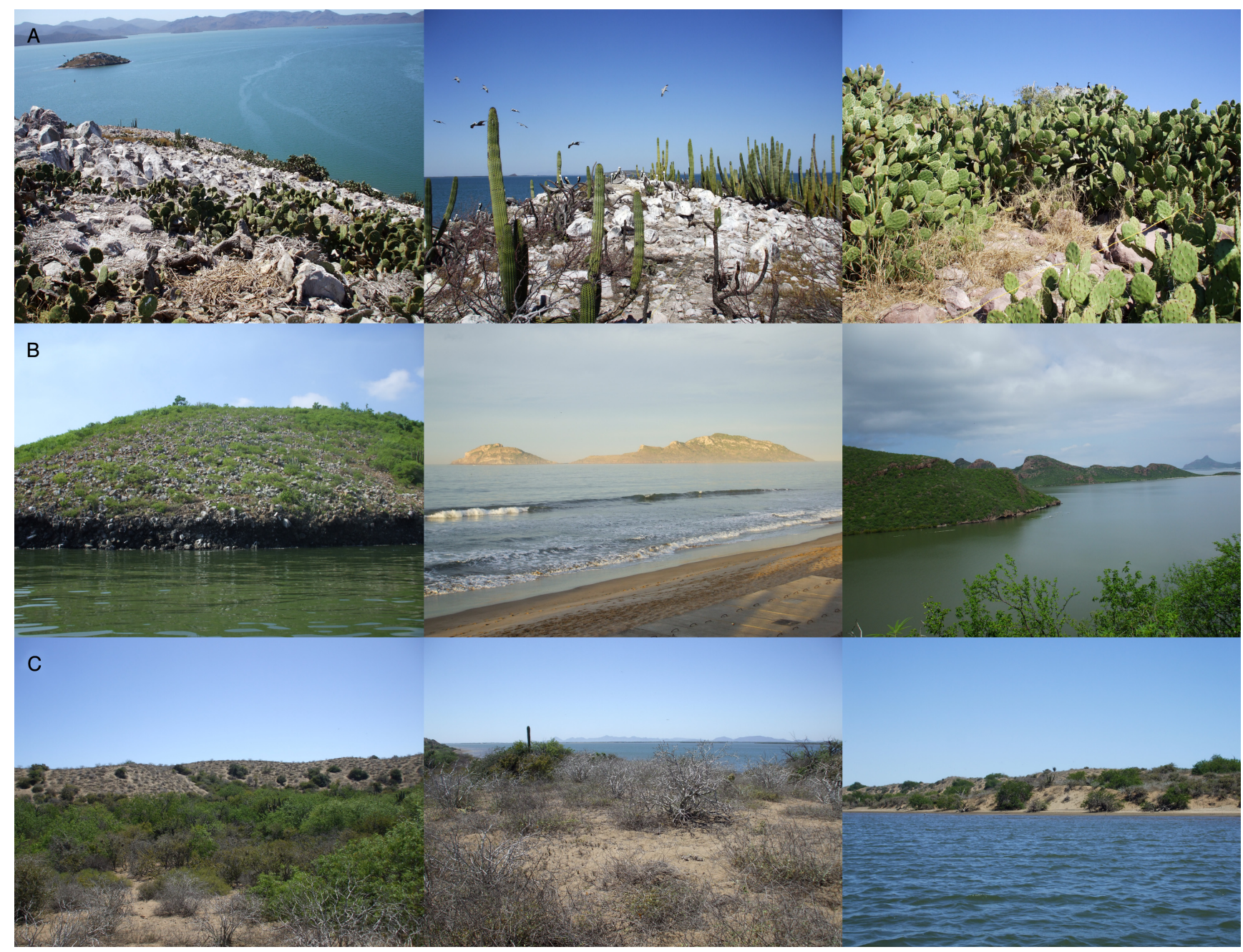

Figura 6. Panorámica de algunas islas de la costa de Sinaloa. A) Islas rocosas que proveen sitios favorables para la reproducción, anidación y percha de aves marinas, B) Islas de origen volcánico con alto porcentaje de piedras y C) Islas arenosas con nutrimentos limitados.

cos y muy secos de otros países (Arshad et al., 2008; Salama et al., 2013; Abd El-Ghani et al., 2014).

Las altas concentraciones de nitratos, fosfatos y $\mathrm{pH}$ ácido que existen en algunas islas de la entidad debido al aporte de guano producido por aves marinas y terrestres (Figura $6 \mathrm{~A})$, mantienen menor riqueza de especies vegetales; lo anterior ocasiona que sólo persistan especies como Opuntia spp. y Stenocereus alamosensis y las arbóreas Crateva tapia, Ficus petiolaris y Plumeria rubra fo. acutifolia; plantas proveedoras de sitios de percha, anidación y reproducción para las comunuidades aviares. Estos resultados se complementan con estudios realizados en islas de Bahía de Ohuira (Patos y Mazocahui) y Bahía Mazatlán (Lobos, Venados y Pájaros). En las islas de la Bahía de Ohuira hay 42 especies más con respecto a las islas de la Bahía de Mazatlán, en una superficie semejante (Reyes-Olivas, 2002; Reyes-Olivas et al., 2008). En otra comparación, la isla Patos alberga numerosos individuos de aves marinas (Guevara-Estrada, 2006) que incrementan las concentraciones de nitrógeno, fósforo y potasio, en comparación con Mazocahui, una isla carente de éste enriquecimiento biogeoquímico, situación que contribuiría a explicar la baja riqueza vegetal de la isla Patos (Reyes-Olivas et al., 2008). En la segunda bahía, la isla Venados tiene 70 especies más que la Pájaros (Flores-Campaña et al., 1996; Vega-Aviña et al., 2001), pero el número de especies en Lobos y Pájaros es semejante; el contraste se da en la superficie del área, debido a que Pájaros es 35.5 ha más grande (Vega-Aviña et al., 2001). La variación notoria en el número de especies en las tres islas, es que en Pájaros se tienen importantes áreas de percha, anidación y reproducción de aves marinas como la garza gris y el pelícano café (Vega-Aviña et al., 2001), lo que incrementa, de manera considerable, las concentraciones de nitratos (3,042.64 ppm), al mismo tiempo reducen el pH del suelo a niveles ácidos (4.8); en cambio, en las dos islas restantes, no hay comunidades aviares, las concentraciones de nitratos son normales (18.95 ppm para 
Venados y 13.00 ppm en Lobos) y aún cuando las concentraciones de fosfatos son altas $(\mu=116.5 \mathrm{ppm}$ y $\mu=113.98$ ppm, de manera respectiva), el pH es menos ácido (5.70 y 5.90 en Venados y Lobos, respectivamente), lo que permite la existencia de mayor riqueza florística. También, el efecto negativo de los ingresos de guano sobre la riqueza vegetal se ha demostrado en islas del golfo de California, debido a que el alto contenido de nitrógeno, fósforo y $\mathrm{pH}$ ácido registrado en los suelos, limitan el crecimiento de plantas (Sánchez-Piñero y Polis, 2000; Wait et al., 2005; Velarde et al., 2014).

La variable topográfica identificada como pendiente, es importante en el área de estudio, también las variables edáficas como cobertura de rocas, profundidad del suelo y porcentajes de arena y limo, debido a que ejercen influencia en la distribución y abundancia de las especies mediante la existencia de sitios seguros, como se ha informado en otras localidades secas o muy secas del país (Huerta-Martínez y García-Moya, 2004; Huerta-Martínez et al., 2004). La topografía interviene en los procesos que limitan la distribución y abundancia de las plantas en varios sentidos. Por ejemplo, Milchunas y Noy-Meir (2002) demostraron que los refugios geológicos, además de ser una restricción física para herbívoros, modifican otras variables como la profundidad del suelo, la humedad debida al escurrimiento, la temperatura, etc., estas variables son diferentes en un área rocosa que en una zona sin rocas. El $75 \%$ de los sistemas insulares analizados en este trabajo se destacan por tener alta cobertura rocosa (Figura 6B) que promedia $57.4 \%$ con variación de $86 \%$ (Bledos) al $20.5 \%$ (Cardo). Investigaciones como las de Parker (1991), Nobel et al. (1992), Peters et al. (2008), López et al. (2009) y Bárcenas-Argüello et al. (2010) describen la relación de las rocas con las propiedades del suelo y el crecimiento de las plantas, debido a que mientras más grandes son las rocas, éstas mejoran el efecto protector para las plantas, por reducción de la radiación solar directa, amortiguamiento de temperaturas extremas del suelo, reducción de la evaporación y canalización del agua a las raíces. Cactáceas y otras especies de ambientes secos del noroeste de México, se distribuyen en micrositios sin suelo, por ejemplo, acantilados, cavidades o fisuras en las rocas y flujos de lava antiguos (Bashan et al., 2002; López et al., 2009; Bárcenas-Argüello et al., 2010). También en la vegetación costera se ha resaltado la importancia biológica de las rocas en la demografía y en la estructura de la comunidad vegetal, en lo particular en las cactáceas, debido a que intervienen en la formación de reservorios de semillas (ValdezManzanares, 2009), propician condiciones ambientales que favorecen la germinación de semillas (Sánchez-Soto et al., 2005, 2010), incrementan la protección de plántulas contra herbívoros (Lugo-García, 2005) y determinan patrones espaciales (Reyes-Olivas et al., 2002).

La estructura y composición de las comunidades vegetales en las islas de barra de la costa de Sinaloa (Figura 6C), entre ellas, Altamura, Iguanas, Saliaca y Talchichilte, están relacionadas con el alto porcentaje de arena (83\%), gran profundidad del suelo $(70 \mathrm{~cm}$ ) y limitación de nutrimentos. En este sentido, Castillo y Moreno-Casasola (1998) destacan que las costas arenosas tienen un sustrato móvil, partículas que varían de tamaño, topografía heterogénea con baja capacidad de retención de humedad y limitación de nutrimentos, características que demarcan los agrupamientos particulares de especies. En lo general, la distribución de la vegetación en dunas costeras a partir de la línea de la costa, inicia con la zona de plantas pioneras en las dunas embrionarias, hasta la ocupación por arbustivas y arbóreas en las dunas estabilizadas (Moreno-Casasola y Espejel, 1986; Romero-López et al., 2006). Altamura y Talchichilte presentan una distribución semejante, teniendo una secuencia de vegetación de dunas, bosque de manglar, halófitas y bosque espinoso, para Altamura; y de manglar, vegetación halófita y bosque espinoso para Talchichilte (Flores-Campaña et al., 2003). En dunas costeras de islas de barra, existe una correlación entre gradientes de vegetación y factores ambientales como clima, suelo, viento, aspersión salina, disponibilidad de agua dulce y de nutrimentos (Lortie y Cushman, 2007; Kim y Yu, 2009; Torres et al., 2010).

\section{Conclusiones}

Se demuestra que los factores del medio que limitan la distribución de especies perennes en las 16 islas consideradas en esta investigación son topográficos y edáficos. Mediante el análisis multivariante se infiere el efecto de cobertura de rocas, pendiente, profundidad del suelo, porcentajes de arena, limo, $\mathrm{pH}$ y nitratos, en tres conjuntos de islas: (1) Sistemas insulares: Alta Blanca, Bledos, Patos y Pájaros que reciben aportes de guano de aves marinas, y cuya consecuencia tiende a ser la disminución de la riqueza específica, (2) Las islas de origen volcánico, rocosas, suelo de textura franco arenosa, con profundidad de 2 a $15 \mathrm{~cm}$ y disponibilidad de nutrimentos, principalmente fosfatos y materia orgánica que facilita la formación de sitios apropiados para la distribución de especies y (3) las islas arenosas, con suelos profundos de $70 \mathrm{~cm}, 82.6 \%$ de arena y nutrimentos limitados.

\section{Agradecimientos}

Se agradece al Consejo Nacional de Ciencia y Tecnología (CONACyT) por la beca otorgada (No. 98895) al primer autor para la realización de estudios doctorales en el Posgrado de Botánica del Colegio de Postgraduados y a Fondos Mixtos de CONACyT-Gobierno de Sinaloa, por el financiamiento de la investigación (SIN-2006-CO1-37439). También se agradece a los revisores anónimos y cuerpo editorial de la revista, quienes aportaron importantes observaciones para la mejora del manuscrito. 


\section{Literatura citada}

Abd El-Ghani M., Soliman A. y Abd El-Fattah R. 2014. Spatial distribution and soil characteristics of the vegetation associated with common succulent plant in Egypt. Turkish Journal of Botany 38:550-565.

Aguado-Santacruz G.A. y García-Moya E. 1998. Environmental factors and community dynamics at the southernmost part of the North American Graminetum: I. On the contribution of climatic factors to temporal variation in species composition. Plant Ecology 135:13-29.

Aguado-Santacruz G.A., García-Moya E., Velasco-González C. y Flores-Flores J.L. 1996. Importancia de los elementos climáticos en la variación florística temporal de pastizales semidesérticos. Acta Botanica Mexicana 35:65-81.

Arshad M., Anwar-Ul-Hussan, Ashraf M.Y., Noureen S. y Moazzam M. 2008. Edaphic factors and distribution of vegetation in the Cholistan Desert, Pakistan. Pakistan Journal of Botany 40:1923-1931.

Bárcenas-Argüello M.L., Gutiérrez-Castorena M.del C., Terrazas T. y López-Mata L. 2010. Rock-soil preferences of three $C e$ phalocereus (Cactaceae) species of tropical dry forests. Soil Science Society of America Journal 74:1374-1382.

Bashan Y., Li C.Y., Lebsky V.K., Moreno M. y De-Bashan L.E. 2002. Primary colonization of volcanic rocks by plants in arid Baja California, Mexico. Plant Biology 4:392-402.

Bremner J.M. 1965. Inorganic forms of nitrogen. In: Black C.A. Ed. Methods of Soil Analysis, Part 2. pp. 1179-1237, Agronomy Monographs 9. American Society of Agronomy, Madison.

Canfield R.H. 1941. Application of the line interception method in sampling range vegetation. Journal of Forestry 39:388-394.

CONAGUA [Comisión Nacional del Agua]. 2014. Estaciones Climatológicas. <http://smn.conagua.gob.mx/climatologia/normales/estacion/EstacionesClimatologicas.kmz> (Consultado 28 de diciembre, 2014).

Castillo S.A. y Moreno-Casasola P. 1998. Análisis de la flora de dunas costeras del litoral Atlántico de México. Acta Botanica Mexicana 45:55-80.

CSTPA [Council on Soil Testing and Plant Analysis]. 1980. Handbook on Reference Methods for Soil Testing. University of Georgia, Athens.

Day P.R. 1965. Particle fractionation and particle-size analysis. En: Black C.A., Evans D.D., White J.L., Ensminger L.E. y Clark F.E. Eds. Methods of Soil Analysis: Part 1. Physical and Mineralogical Properties, Including Statistics of Measurement and Sampling, pp. 545-567, Agronomy Monographs 9. American Society of Agronomy, Madison.

Díaz [HT1]J.S. 2008. Diversidad florística y estructura de la vegetación de las islas de los sistemas lagunares Navachiste y Macapule, del norte de Sinaloa. Tesis de maestría, Centro Interdisciplinario de Investigación para el Desarrollo Integral Regional, Unidad Sinaloa-IPN, Guasave, Sinaloa, México. 132 pp.

Duarte M.C., Rego F. y Moreira I. 2005. Distribution patterns of plant communities on Santiago Island, Cape Verde. Journal of Vegetation Science 16:283-292.

Fernández-Palacios J.M. y de Nicolás J.P. 1995. Altitudinal pattern of vegetation variation on Tenerife. Journal of Vegetation Science 6:183-190.

Flores-Campaña L.M., Ortiz-Arellano M.A. y Arzola-González J.F. 2003. Islas e islotes de Sinaloa. En: Cifuentes-Lemus J.L. y
Gaxiola-López J. Eds. Atlas de los Ecosistemas de Sinaloa, pp. 111-126, El Colegio de Sinaloa. México.

Flores-Campaña L.M., Vega-Aviña R., Benítez-Pardo D. y Hernández-Álvarez F. 1996. Flora de la isla Venados de la bahía Mazatlán, Sinaloa, México. Anales del Instituto de Biología de la Universidad Nacional Autónoma de México, Serie Botánica 67:283-301.

García E. 1988. Modificaciones al Sistema de Clasificación Climática de Köppen Para Adaptarlo a las Condiciones Particulares de la República Mexicana. Offset Larios. México D.F.

Geiger R. 1966. The Climate near the Ground. Harvard University Press, Cambridge.

Gobierno del Estado de Sinaloa. 2007. Plan Estatal de Desarrollo Urbano del Estado de Sinaloa. Secretaría de Desarrollo Social y Sustentable, Culiacán.

Guevara-Estrada A. 2006. Riqueza y abundancia de aves en isla Patos, Bahía de Ohuira, Sinaloa, México, 2003. Tesis de licenciatura, Universidad de Occidente, Unidad Los Mochis, Los Mochis, Sinaloa, México. 40 pp.

Hill M.O. 1979. Decorana: A FORTRAN Program for Detrended Correspondence Analysis and Reciprocal Averaging. Cornell University, Ithaca.

Hill M.O. y Gauch Jr. H.G. 1980. Detrended correspondence analysis, an improved ordination technique. Vegetatio 42:47-58.

Huerta-Martínez F.M. y García-Moya E. 2004. Diversidad de especies perennes y su relación con el ambiente en un área semiárida del centro de México: Implicaciones para su conservación. Interciencia 29:435-441.

Huerta-Martínez F.M., Vázquez-García J.A., García-Moya E., López-Mata L. y Vaquera-Huerta H. 2004. Vegetation ordination at the southern Chihuahuan Desert (San Luis Potosi, Mexico). Plant Ecology 174:79-87.

Kim D. y Yu K.B. 2009. A conceptual model of coastal dune ecology synthesizing spatial gradients of vegetation, soil, and geomorphology. Plant Ecology 202:135-148.

López B.R, Bashan Y., Bacilio M. y De la Cruz-Agüero G. 2009. Rock-colonizing plants: abundance of the endemic cactus $M a$ mmillaria fraileana related to rock type in the southern Sonoran Desert. Plant Ecology 201:575-588.

Lortie C.J. y Cushman J.H. 2007. Effects of a directional abiotic gradient on plant community dynamics and invasion in a coastal dune system. Journal of Ecology 95:468-481.

Lugo-García G.A. 2005. Mortalidad de cactáceas causada por herbívoros en una isla de Topolobampo, Sinaloa. Tesis de licenciatura, Universidad Autónoma de Sinaloa, Juan José Ríos, Ahome, Sinaloa. México. 61 pp.

McAuliffe J.R. 1994. Landscape evolution, soil formation, and ecological patterns and processes in Sonoran Desert bajadas. Ecological Monographs 64:112-148.

McCune B. y Grace J.B. 2002. Analysis of Ecological Communities. MjM Software, Gleneden Beach.

McCune B. y Mefford M.J. 2011. PC-ORD for Windows. Multivariate Analysis or Ecological Data V. 6.0. MjM Software, Gleneden Beach.

Milchunas D.G. y Noy-Meir I. 2002. Grazing refuges, external avoidance of herbivory and plant diversity. Oikos 99:113-130.

Moreno-Casasola P. y Espejel I. 1986. Classification and ordination of coastal sand dune vegetation along the Gulf and Caribbean Sea of Mexico. Vegetatio 66:147-182.

Nobel P.S., Miller P.M. y Graham E.A. 1992. Influence of rocks on 
soil temperature, soil water potential, and rooting patterns for desert succulents. Oecologia 92:90-96.

Oke T.R. 1987. Boundary layer climates. Methuen, Londres.

Otto R., Fernández-Palacios J.M. y Krüsi B.O. 2001. Variation in species composition and vegetation structure of succulent scrub on Tenerife in relation to environmental variation. Journal of Vegetation Science 12:237-248.

Palmer M.W. 1993. Putting things in even better order: the advantages of canonical correspondence analysis. Ecology 74:22152230.

Parker K.C. 1991. Topography, substrate, and vegetation patter$\mathrm{ns}$ in the northern Sonoran Desert. Journal of Biogeography 18:151-163.

Pausas J.G. y Austin M.P. 2001. Patterns of plant species richness in relation to different environments: an appraisal. Journal of Vegetation Science 12:153-166.

Peters E.M. Martorell C. y Ezcurra E. 2008. Nurse rocks are more important than nurse plants in determining the distribution and establishment of globose cacti (Mammillaria) in the Tehuacan Valley, Mexico. Journal of Arid Environments 72:593-601.

Reyes-Olivas A. 2002. Patrones espaciales de cactáceas en el desierto costero de Topolobampo. Tesis doctoral, Colegio de Postgraduados, Montecillo, Texcoco, Estado de México, México. 184 pp.

Reyes-Olivas A., Apodaca-Ovalle V., Cota-Sánchez J.H. y Casillas-Álvarez P. 2008. Relación del suelo y la topografía con la diversidad y la estructura de la vegetación insular en el desierto costero de Sinaloa, México. En: Flores-Campaña L.M. Ed. Estudios de las Islas del Golfo de California, pp. 53-66, Universidad Autónoma de Sinaloa-Gobierno del Estado de SinaloaConsejo Nacional de Ciencia y Tecnología, México D.F.

Reyes-Olivas A., García-Moya E. y López-Mata L. 2002. Cacti-shrub interactions in the coastal desert of northern Sinaloa, Mexico. Journal of Arid Environments 52:431-445.

Richards L.A. 1954. Diagnosis and Improvement of Saline and Alkali Soils. Agriculture Handbook No. 60, United States Department of Agriculture, Washington D.C.

Romero-López B.E., León-de la Luz J.L., Pérez-Navarro J.J. y De la Cruz-Agüero G. 2006. Estructura y composición de la vegetación de la barra costera El Mogote, Baja California Sur, México. Boletín de la Sociedad Botánica de México 79:21-32.

Rzedowski J. 1978. Vegetación de México. Editorial Limusa S.A, México D.F.

Salama F., Abd El-Ghani M. y El-Tayeh N. 2013. Vegetation and soil relationships in the inland wadi ecosystem of central Eastern Desert, Egypt. Turkish Journal of Botany 37:489-498.

Sánchez-Piñero F. y Polis G.A. 2000. Bottom-up dynamics of allochthonous input: direct and indirect effects of seabirds on islands. Ecology 81:3117-3132.
Sánchez-Soto B.H., García-Moya E., Terrazas T. y Reyes-Olivas A. 2005. Efecto de la hidratación discontinua sobre la germinación de tres cactáceas del desierto costero de Topolobampo, Ahome, Sinaloa. Cactáceas y Suculentas Mexicanas 50:4-14.

Sánchez-Soto B., Reyes-Olivas A., García-Moya E. y Terrazas T. 2010. Germinación de tres cactáceas que habitan la región costera del noroeste de México. Interciencia 35:299-305.

SAS Institute Inc. 2009. SAS/STAT User's Guide Release 9.2. SAS Institute, Cary.

Shreve F. y Wiggins I.L. 1964. Vegetation and Flora of the Sonoran Desert. Vol. I y II. Stanford University Press, Palo Alto.

Ter Braak, C. J. F. 1986. Canonical correspondence analysis, a new eigenvector technique for multivariate direct gradient analysis. Ecology 67:1167-1179.

Ter Braak C.J.F. y Prentice I.C. 1988. A theory of gradient analysis. Advances in Ecological Research 18:271-317.

Ter Braak C.J.F. y Šmilauer P. 2002. CANOCO Reference Manual and CanoDraw for Windows User's Guide: Software for Canonical Community Ordination (version 4.5).Microcomputer Power, Ithaca. Disponible en: <www.canoco.com>

Torres W., Méndez M., Dorantes A. y Durán R. 2010. Estructura, composición y diversidad del matorral de duna costera en el litoral Yucateco. Boletín de la Sociedad Botánica de México 86:37-51.

Valdez-Manzanares A.E. 2009. Distribución y consumo de semillas de cactáceas en el desierto costero de Topolobampo. Tesis de licenciatura, Universidad Autónoma de Sinaloa. Juan José Ríos, Ahome, Sinaloa, México. 74 pp.

Valverde P.L., Zavala-Hurtado J.A., Montaña C. y Ezcurra E. 1996. Numerical analyses of vegetation based on environmental relationships in the southern Chihuahuan Desert. The Southwestern Naturalist 41:424-433.

Vega-Aviña R., Benítez-Pardo D., Flores-Campaña L.M. y Hernández-Álvarez F. 2001. Vegetación y flora de la isla Pájaros e isla Lobos de la bahía de Mazatlán, Sinaloa. Listados Florísticos de México 21:7-19.

Velarde E., Wilder B.T., Felger R.S. y Ezcurra E. 2014. Floristic diversity and dynamics of Isla Rasa, Gulf of California - a globally important seabird island. Botanical Sciences 92:89-101.

Wait D.A., Aubrey D.P. y Anderson W.B. 2005. Seabird guano influences on desert islands: soil chemistry and herbaceous species richness and productivity. Journal of Arid Environments 60:681-695.

Walkley A. y Black I.A. 1934. An examination of the Degtjareff method and a proposed modification of the chromic matter and a proposed modification of the chromic acid titration method. Soil Science 37:29-38.

Wiggins I.L. 1980. Flora of Baja California. Stanford University Press, Palo Alto.

Recibido: 28 de agosto de 2014

Aceptado: 26 de enero de 2015 